\title{
Materials Science of Supported Lipid Membranes
}

\section{Atul N. Parikh and Jay T. Groves, Guest Editors}

\begin{abstract}
Supported membranes represent an elegant route to designing well-defined fluid interfaces which mimic many physical-chemical properties of biological membranes. Recent years have witnessed rapid growth in the applications of physical and materials science approaches in understanding and controlling lipid membranes. Applying these approaches is enabling the determination of their structure-dynamics-function relations and allowing the design of membrane-mimetic devices. The collection of articles presented in this issue of MRS Bulletin illustrates the breadth of activity in this growing partnership between materials science and biophysics. Together, these articles highlight some of the key challenges of cellular membranes and exemplify their utility in fundamental biophysical studies and technological applications. The topics covered also confirm the importance of lipid membranes as an exciting example of soft condensed matter. We hope that this issue will serve readers by highlighting the intellectual scope and emerging opportunities in this highly interdisciplinary area of materials research.
\end{abstract}

Keywords: biological, biomimetic, cellular.

Lipid bilayer membranes are the universal material of choice for defining and controlling cellular organization in living systems. They are a major constituent of the biological membranes that serve as the outer boundary of cells and organelles. ${ }^{1}$ As such, they serve as a means to compartmentalize, juxtapose, regulate, and generally mediate biomolecular interactions. The astounding complexity of life is intimately associated with the diverse physical-chemical properties of these membranes, ${ }^{2}$ which include two-dimensional fluidity, material elasticity, thermal fluctuations, chemical diversity, and rich phase behavior. ${ }^{3}$ In this regard, developing a materials-science-based understanding of lipid membranes is an exciting endeavor.

Traditional approaches to materials synthesis have largely relied on uniform, equilibrated phases leading to static "condensed matter" structures, for example, monolithic single crystals. Over the past several decades, these approaches have led to the development of a wide range of technologically useful materials including semiconducting, ferroelectric, nonlinear optical, superconducting, and piezoelectric materials. Important as they are, the range of functions in these materials is constrained by their static structure. In contrast, lipid bilayer membranes exploit their chemical heterogeneity, phase behavior, and dynamics to produce an impressive set of time-dependent functions for the biological membrane. Examples include stimuli-induced protein clustering, lipid reorganization, and topographical changes that regulate broad classes of biological functions of membranes ${ }^{4,5}$ including signaling, molecular recognition, and transport. This spatio-temporal mode of lipid organization, or dynamic self-assembly, in biological membranes ${ }^{6}$ exemplifies a major shift in emphasis from thermodynamic to kinetic regimes. Here, equilibrium structures (global free-energy minima) are replaced by higher-order organizational states representing facile transitions between various local metastable free-energy minima of different structures. Examples of synthetic materials deliberately fabricated using such a dynamic self-assembly approach are rare, reflecting the current lack of a fundamental understanding of such processes. We assert that the creation of new materials capable of performing advanced technological functions, for example, complex and cooperative processes for memory, self-replication, and self-repair, will ultimately rely upon understanding and controlling dynamic self-assembly in both natural and synthetic systems. We emphasize that understanding the interrelations between structure, assembly, dynamics, and function in lipid membranes contributes toward these broad objectives of contemporary materials science.

Furthermore, there are many direct and practical benefits of learning to devise synthetic materials that mimic the key properties of cellular membranes. Such materials may provide new classes of biosensors, diagnostic tools, biocompatible materials, and high-throughput characterization platforms for rapid and early detection of interactions between cells and their environment. Examples include sensors and assays for detecting toxins and pathogens (e.g., in national security applications), air and water quality monitoring, and drug screening platforms. They also promise tools for life science research, for example, in developing a molecular-level understanding of cell surface mechanisms during cellular homeostasis and diseases.

Within this framework, one approach that is proving powerful involves interfacing lipid bilayer membranes to solid substrates via self-assembly and directed self-assembly methods. These membrane-mimicking architectures are collectively referred to as supported membranes $^{7}$ (Figure 1).

Supported membranes are typically formed at the solid-liquid interface when vesicular microphases of lipids spontaneously rupture and spread on hydrophilic surfaces (Figures 1a and 1b). Alternative strategies employ Langmuir-Blodgett troughs to successively transfer two lipidic monolayers from the air-water interface onto planar surfaces ${ }^{3,8}$ (Figure 1c). Recent studies reveal that single lipid bilayers can also form when dried lipids stamped onto a hydrophilic surface experience hydration. ${ }^{9}$ In all of these cases, single lipid bilayers are separated from the substrate surface through an intervening layer of hydration water (dark-blue layer in Figure 1d, variously estimated at 6-15 $\AA$ in thickness at bilayer-silica interfaces) and exhibit two-dimensional contiguity and fluidity resembling that of lipid membranes of vesicles and living cells. ${ }^{10}$ Each of the two lipid layers in the lipid bilayer is called a "leaflet." In cells, the outer leaflet is in contact with the extracellular matrix, and the inner leaflet faces the cell cytoplasm. The precursor phases in these 

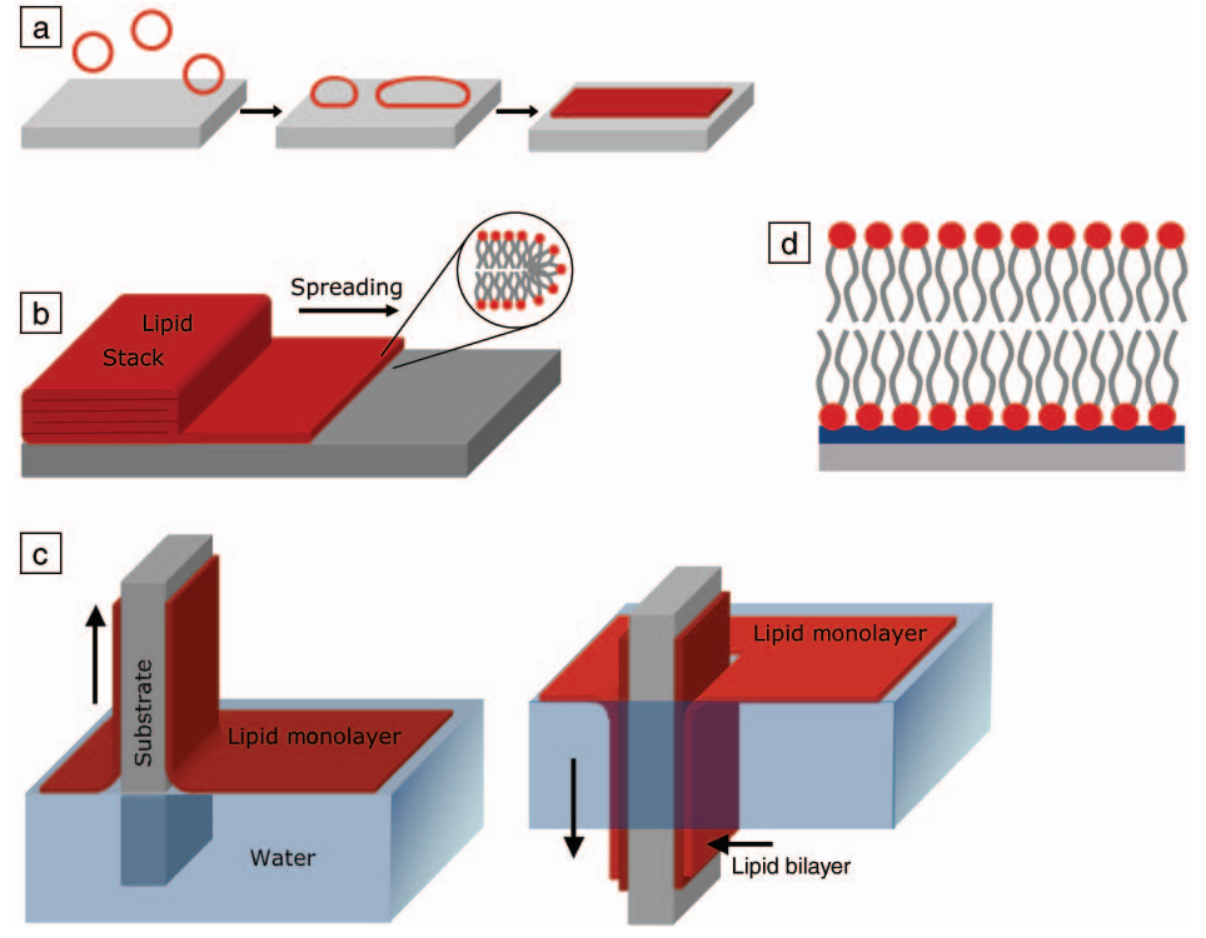

Figure 1. Schematic illustration of basic supported-membrane formation processes. (a) Vesicles adsorb, fuse, and rupture at solid surfaces; (b) lipid stacks spontaneously spread upon hydration; and (c) two successive transfers of Langmuir-Blodgett monolayers deposit pre-equilibrated lipid mixtures to form (d) single, supported bilayers on hydrophilic substrates. The dark-blue area is the intervening layer of hydration water between the substrate and the lipid.

approaches can consist of purified and synthetically tailored lipids and their complex mixtures in predetermined compositions, providing molecular-level control over their structure, chemical composition, phase state, and lateral fluidity. Moreover, because these constructs interface membrane architecture with solid substrates, they are amenable to a broad suite of surface analytical tools for molecularscale characterization of their structure and dynamics and enable chip-based bioanalytical assays, sensors, and platforms.

We hope that this issue of MRS Bulletin serves to highlight the broad and rapidly evolving intellectual partnership between the materials sciences and membrane biophysics. This partnership underscores an interdisciplinary perspective in at least two ways.

First, it reveals how generating a scientific environment in which fundamental concepts obtained through detailed study of systems in one scientific area can be rapidly applied to generate understanding toward other, traditionally distinct, disciplines. For instance, it illustrates how the supported-membrane-based studies, aimed purportedly at understanding and mimicking biological membranes, provide an experimental test bed for questions in soft condensed matter, interfacial confinement, low-dimensional phases, chemistry in two dimensions, reactiondiffusion systems, and wetting.

Second, it demonstrates how the application of principles, tools, and methodologies developed extensively in one area of science can benefit the development of another, disparate discipline. We emphasize that the two-way traffic between fundamental studies of membranes and soft condensed matter research is rapidly elaborating. The articles presented here merely illustrate, rather than provide a comprehensive survey of, the breadth and the scope of this partnership.

Despite its popularity, the supported membrane configuration experiences many challenges in modeling physical-chemical properties of biological membranes because of the coupling of the bilayer to the rigid substrate surface. This interface suppresses thermally activated membrane fluctuations, limits the incorporation of membrane proteins, induces substrate-electrostatics-driven asymmetry, and hinders the translational mobilities and phase equilibration in bilayers. The article by Tanaka emphasizes these issues and offers a strategy to cushion the membrane-substrate interface via ultrathin polymer supports. He further demonstrates how the use of intercalated polymers may offer a materials route to modeling the generic role of the extracellular matrix and glycocalyx. The term glycocalyx refers to a variety of glycosylated proteins, glycolipids, and polymeric structures that often terminate cell surfaces.

An important structural feature of lipid membranes in biology is their ability to bend. There is a growing recognition that membrane curvature is an active regulator of membrane microphase separation and a concentrator of important biological functions. Sasaki and Stevens emphasize the issue, survey a physical-science-based understanding of the formation of these submembrane structural features, and highlight associated materials issues.

The perspective by Zhang and Granick illustrates the importance of advanced characterization methods in characterizing the lateral dynamics in supported membranes. A long-standing issue, one with many inconsistencies, relates to the issue of complex translational dynamics. Structural, chemical, and phase heterogeneities within membranes exist both statically and dynamically (such as in response to external stimuli, e.g., protein binding). Moreover, these heterogeneities often emerge independently in the two leaflets of the bilayer architecture. Complex dynamics attending such asymmetries requires advanced characterization tools capable of measuring molecular diffusivities at a range of length and time scales, also discriminating the two leaflets.

The article by Wu et al. is focused on the permeability characteristics of cellular membranes. Using supported membranes and lipid monolayers, the authors illustrate how synthetic molecules (e.g., triblock copolymers) can be used to repair damaged membranes. They demonstrate the ability of a class of triblock copolymers to selectively incorporate within damaged or void regions of the membranes. Their study further reveals how cells might rid the polymer from their membranes once the structural integrity of the membrane is restored.

Another long-standing issue in solidsupported lipid bilayers that continues to hamper their practical application in sensing and detection relates to their longterm stability. As Daniel et al. correctly point out, "they lack the resiliency to withstand air exposure and the thermal and mechanical stresses associated with device transport, storage, and continuous use over long periods of time." Their article summarizes several new strategies 
that mimic cellular cytoskeletons, thus enhancing bilayer stability.

The article by Fang et al. summarizes the current state of biomembrane technologies that enable the preservation and parallel display of functionally active membrane targets. This ability promises to deliver much-needed membranemimetic platforms for screening potential drug compounds against their membrane targets (e.g., G-protein coupled receptors, or GPCRs - a pervasive family of transmembrane proteins, targeted by $40-50 \%$ drugs, that transduce a host of extracellular ligand-binding signals into intracellular signals). The overwhelming role of membrane proteins as pharmacological targets put this issue at the center of this technology development.

The collection of review/perspective articles presented in this issue of $M R S$ Bulletin represents a growing body of materials work targeted at understanding and controlling lipid membranes, largely in synthetic settings. These are complex systems that present many challengesmany of which are highlighted within these reviews. Complex as the issues are, there is plenty of room for optimism. Supported membranes have proved remarkably successful in a number of applications, including as a means of creating surrogate cell surfaces for interactions with other living cells. Most visually evocative among these implementations, perhaps, are the immunological synapses that have been induced to form between living T cells and supported membranes. ${ }^{11,12}$

Building on this methodology, the supported membrane configuration provides an avenue to introduce patterns of external stimuli-including inorganic nanostructures, surface patterns, signaling molecules, and oxidative stresses-to cells using their own receptors as liaisons (see Figure 2). These abilities offer promise

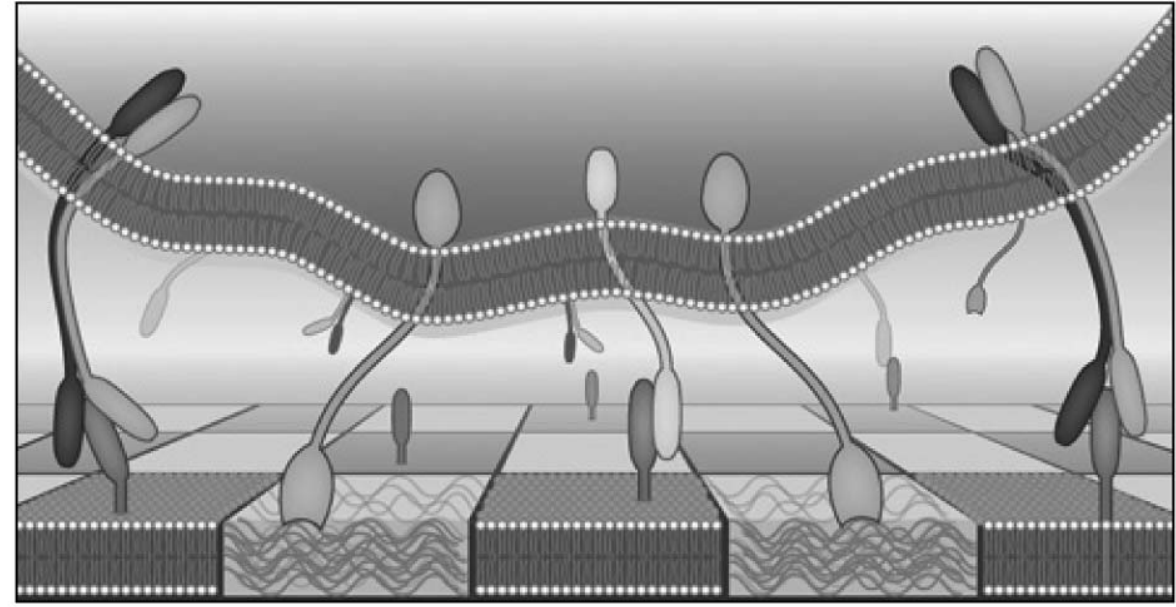

Figure 2. Schematic image of a living cell (at top) interacting with a supported-membranecoated synthetic surface (at bottom). Natural protein signaling molecules may be incorporated into the synthetic membrane, providing specific functionalities with which the living cell may interact.

toward understanding molecular interactions that characterize cellular homeostasis and disease. Several of these exciting applications are already paying dividends, and with greater development of the system from a controlled materials perspective, we can anticipate much more yet to come.

\section{Acknowledgments}

This work is supported by grants from Basic Energy Sciences, U.S. Department of Energy, and a partnership grant between J.T. Groves and A.N. Parikh from the National Science Foundation Center for Biophotonics Science and Technology at the University of California, Davis.

\section{References}

1. J. Darnell, H. Lodish, and D. Baltimore, Molecular Cell Biology (W.H. Freeman, San Francisco, 1990).
2. D.W. Deamer, Origins Life 17 (1986) p. 3.

3. R. Lipowsky and E. Sackmann, eds., Structure and Dynamics of Membranes (NorthHolland, Amsterdam, 1995).

4. K. Simons and D. Toomre, Nat. Rev. Mol. Cell Biol. 1 (2000) p. 31.

5. S. Damjanovich, R. Gaspar, and C. Pieri, Q. Rev. Biophys. 30 (1997) p. 67

6. G. Vereb, J. Szollosi, J. Matko, P. Nagy, T. Farkas, L. Vigh, L. Matyus, T.A. Waldmann, and S. Damjanovich, Proc. Natl. Acad. Sci. USA 100 (2003) p. 8053.

7. E. Sackmann, Science 271 (1996) p. 43.

8. L.K. Tamm and H.M. McConnell, Biophys. J. 47 (1985) p. 105.

9. J. Nissen, K. Jacobs, and J.O. Radler, Phys Rev. Lett. 86 (2001) p. 1904.

10. J.T. Groves and S.G. Boxer, Acc. Chem. Res. 35 (3) (2002) p. 149

11. A.A. Brian and H.M. McConnell, Proc. Natl. Acad. Sci. USA-Bio. Sci. 81 (1984) p. 6159.

12. A. Grakoui, S.K. Bromley, C. Sumen, M.M. Davis, A.S. Shaw, P.M. Allen, and M.L. Dustin Science 285 (1999) p. 221.
Atul N. Parikh, Guest Editor for this issue of MRS Bulletin, is an associate professor of applied science at the University of California, Davis. He received his BChemEng degree from the University of Bombay and his PhD degree from the Materials Science and Engineering Department at the Pennsylvania State University. Previously, he was a postdoctoral scholar and then a technical staff member in the chemical science and bioscience divisions at Los Alamos National Laboratory from 1996 to 2001.

His present research interests include molecular and mesoscale selfassembly, physical chemistry of surfaces, phase transitions and cooperative processes, nanoscale phenomena, and biomolecular spectroscopy spanning soft condensed matter, membrane biophysics, and cell biology.
Parikh can be reached at the University of California, Davis, Department of Applied Science, 3007 Engineering III, One Shields Avenue, Davis, CA 95616-8254 USA; tel. 530-754-7055, fax 530752-2444, and e-mail anparikh@ucdavis.edu.

Jay T. Groves, Guest Editor for this issue of MRS Bulletin, has been a faculty member of the Chemistry Department

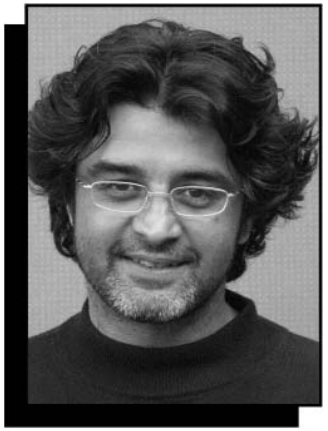

Atul N. Parikh

at the University of California, Berkeley, since 2001. He earned

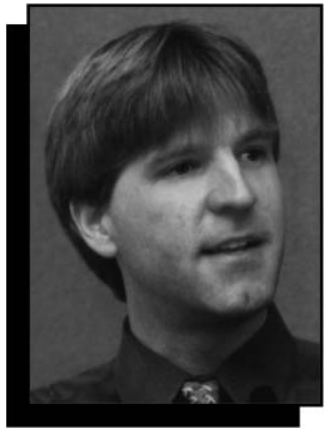

Jay T. Groves

his BS degree in physics and chemistry at Tufts University, followed by 


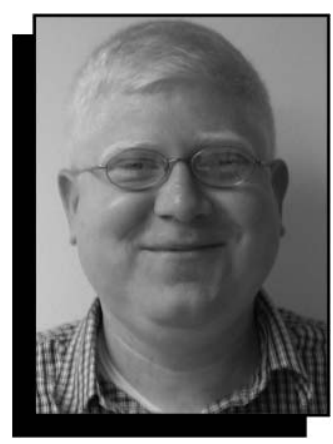

Fernando Albertorio

his $\mathrm{PhD}$ degree from Stanford University in biophysics, working with Steve Boxer and Harden McConnell. During his PhD work, Groves first began to work with supported membranes and developed strategies for patterning supported membranes on surfaces. After graduation, he spent a year as a visiting scholar at Academia Sinica in Taipei, Taiwan; he then took an independent position as a division director's fellow at the Lawrence Berkeley National Laboratory.

His research interests involve the role of spatial organization as a mechanism of regulating the chemical reactions of life. In recent work, his group has introduced the idea of using spatially patterned supported membranes to generate "spatial mutations" of living signaltransduction networks.

Groves can be reached at the University of California, Berkeley, Chemistry Department, 105 Lewis Hall, Berkeley, CA 94720-1460 USA; tel. 510-643-0186, fax 510486-6059, and e-mail jtgroves@lbl.gov.

Fernando Albertorio is a graduate student in the Department of Chemistry at Texas A\&M University, working with Paul S. Cremer. He

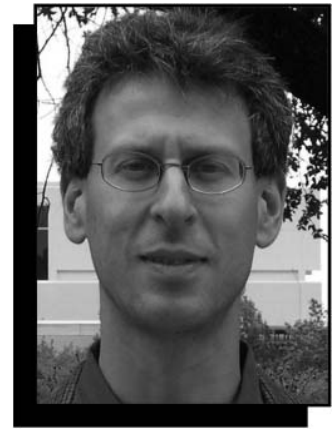

Paul S. Cremer

received his BS degree in chemistry in 1998 from the Pontifical Catholic University of Puerto Rico. Since joining the Cremer group in 2001, his research has focused on developing biomimetic sensors using supported lipopolymer membranes. His interests are in the areas of nanotechnology, bioMEMs, and membrane biophysics.

Albertorio can be reached at Texas A\&M University, Department of Chemistry, 3255

TAMU, College Station, TX 77843 USA; e-mail albertorio@mail.chem. tamu.edu.

Paul S. Cremer is a professor of chemistry at Texas A\&M University. $\mathrm{He}$ received his $\mathrm{PhD}$ degree in chemistry from the University of California, Berkeley, in 1996 and spent two years as a postdoctoral fellow at Stanford University before beginning his academic career at Texas A\&M in 1998. His research interests fall in the field of biological interfaces, physical chemistry, and biotechnology. His laboratory currently investigates problems ranging from protein folding and biofouling to interfacial water structure and multivalency.

Cremer can be reached at Texas A\&M University, Department

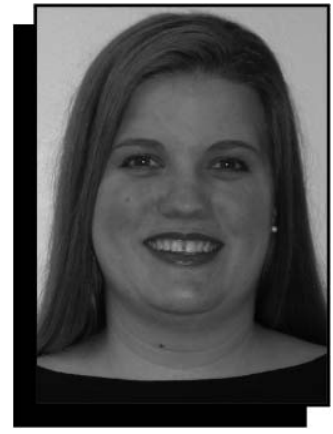

Susan Daniel

of Chemistry, 3255

TAMU, College Station, TX 77845 USA; tel. 979862-1200, fax 979-845-

7561, and e-mail cremer@ mail.chem.tamu.edu.

Susan Daniel is a postdoctoral research associate at Texas A\&M University. She received her PhD degree in chemical engineering from Lehigh University in 2005. She then moved to Texas A\&M to work with Paul S. Cremer in the Department of Chemistry. Since joining Cremer's group, her research has focused on solid-supported lipid bilayers for biosensing applications, studies of protein interactions in bilayers, and nanopore technology.

Daniel can be reached at Texas A\&M University, Department of Chemistry, MS 3255, College Station, TX 77845 USA; tel. 979-8457638 and e-mail sdaniel@ mail.chem.tamu.edu.

Ye Fang is a research associate at Corning Inc. and a visiting professor at the Graduate School of Chemistry and Chemical Engineering, Chinese Academy of Sciences. He received his BS degree in chemistry in 1989 from Hubei University, his MS degree in physical chemistry in 1992 from Wuhuan University, and his $\mathrm{PhD}$

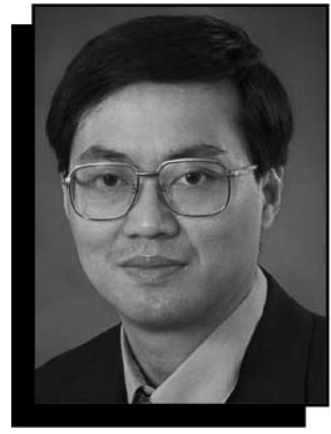

Ye Fang

degree in biophysical chemistry in 1995 from the Institute of Chemistry at the Chinese Academy of Sciences in Beijing. He then carried out postdoctoral research in biophysics at the University of Vermont from 1995 to 1996 and at the Johns Hopkins University's School of Medicine from 1996 to 2000.

Fang joined Corning as a senior research scientist in 2000. His areas of research include biophysics, nucleic acid chemistry, cell biology, protein microarrays, scanning probe microscopies, and optical biosensors. He has published more 70 papers.

Fang can be reached at Corning Inc., 1 Science Center Drive, SPFR-01-5, Painted Post, NY 14870 USA; tel. 607974-7203 and e-mail fangy2@ corning.com.

Shelli L. Frey is a doctoral student at the University of Chicago. She received her BS in chemistry from Haverford College and her MS degree in chemistry from the University of Chicago. Her research interests are in biophysical chemistry, exploring substrate-lipid interactions in the cell membrane and, more specifically, polymer interactions with model lipid membranes. She has been the recipient of

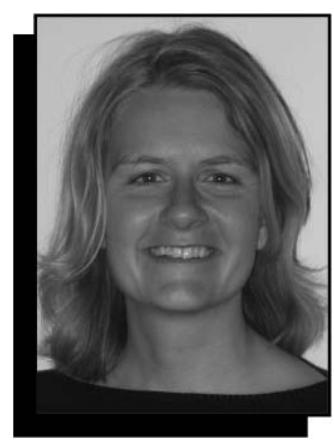

Shelli L. Frey

an NSF graduate research fellowship, a Fulbright fellowship, a Beckman scholarship, and a Goldwater scholarship.

Frey can be reached at the University of Chicago, Department of Chemistry, 5735 S. Ellis Avenue, Chicago, IL 60637 USA; tel. 773834-4036, fax 773-7020805, and e-mail sfrey@uchicago.edu.

Steve Granick is

Founder Professor of Materials, a professor of chemistry, and a professor of physics at the University of Illinois at Urbana-Champaign. He received his BA degree from Princeton University in 1978 and a PhD degree from the University of WisconsinMadison in 1982. He joined the faculty of UIUC in 1985, following postdoctoral research at the Collège de France with P.-G. de Gennes and at the University of Minnesota with Matthew Tirrell. His research interests are in the areas of single-molecule methods, polymers, tribology, complex fluids, and biomaterials. $\mathrm{He}$ has published more than 190 papers on these topics.

A fellow of the American Physical Society, he is chair of the Division of Polymer Physics and associate of the Center for Advanced Study at UIUC. 


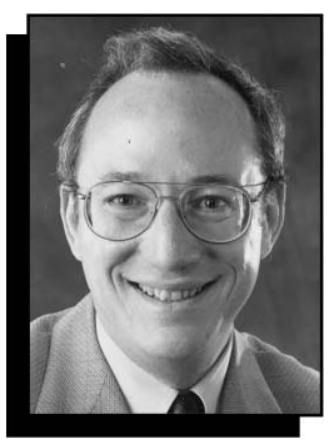

Steve Granick

Granick can be reached at the University of Illinois at Urbana-Champaign, Department of Materials Science and Engineering, 201 MSEB, MC 246, 1304 W. Green Street, Urbana, IL 61801 USA; tel. 217-333-5720, fax 217-244-2278, and e-mail sgranick@ uiuc.edu.

Yulong Hong is a research associate at Corning Inc. He received his BS degree in chemistry in 1987, his MS degree in analytical chemistry in 1990 from Peking University, and his PhD degree in biochemistry in 1996 from City University of New York.

After a year as a postdoctoral researcher in the Biological Chemistry Department at the University of Michigan, he joined the Department of Molecular Neuroscience at ParkeDavid Pharmaceutical Co. in Ann Arbor, Michigan, as a research fellow. He then joined Corning as a senior research scientist in 2000. His areas of research include assay development for highthroughput drug discovery, DNA and protein microarrays, cell biology, molecular biology, parasitology, and yeast genetics. Hong has published more than 20 papers.

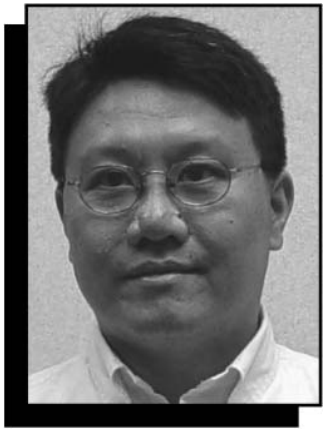

Yulong Hong

Hong can be reached at Corning Inc., 1 Science Center Drive, SPFR-01-5, Painted Post, NY 14870 USA; tel. 607974-1171 and e-mail hongy@corning.com.

Joydeep Lahiri is the research director of biochemical technologies in the Science and Technology Division of Corning Inc. He obtained his bachelor's degree from St. Stephen's College in Delhi, India, his master's degree from the Indian Institute of

Technology in Kanpur, and his doctoral degree in chemistry from

Princeton University. He carried out postdoctoral research at Harvard University. Lahiri then joined Corning in 1999 as a senior research scientist. His research interests include label-free technologies, microarray technologies, and biosurfaces.

Lahiri can be reached at Corning Inc., $1 \mathrm{Sci}-$ ence Center Drive, SPFR-01-5, Painted Post, NY 14870 USA; tel. 607974-9039 and e-mail lahirij@corning.com.

Ka Yee C. Lee is an associate professor in the Department of Chemistry, the Institute for Biophysical Dynamics, and the James Franck Institute at the University of Chicago. She received her ScB degree in

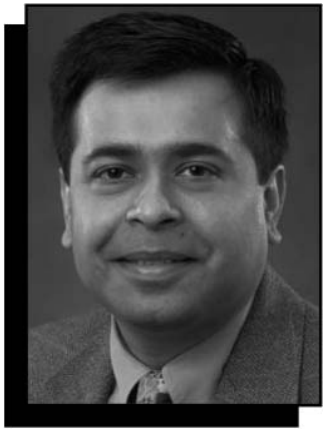

Joydeep Lahiri

electrical engineering from Brown University in 1986, and her MS and PhD degrees in applied physics from Harvard University in 1987 and 1992, respectively. She did her first postdoctoral training in chemistry at Stanford University and her second in chemical engineering at the University of California at Santa Barbara. She joined the faculty of the University of Chicago in 1998.

Her research focuses on the interaction of lipids with proteins or polymers at interfaces, and she has carried out biophysical studies to elucidate the role of membranes in betaamyloid aggregation, the functioning of lung surfactant, the targeting selectivity of antimicrobial peptides, the mechanism of membrane sealing by polymers, and the interaction of cholesterol with lipids in membranes.

Lee has received a Dreyfus New Faculty Award, a March of Dimes Basil O'Connor Starter Scholar Research Award, a Searle Scholar Award, a David and Lucile Packard science and engineering fellowship, the American Health Assistance Foundation's Ruth Salta Junior Investigator Achievement Award in Alzheimer's Disease Research, the Biophysical Society's

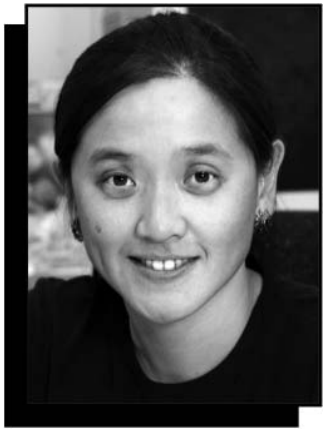

Ka Yee C. Lee

Margaret Oakley Dayhoff Award, and an Alfred P. Sloan fellowship. Lee can be reached at the University of Chicago, Department of Chemistry, 5735 S. Ellis Avenue, Chicago, IL 60637 USA; tel. 773-7027068, fax 773-702-0805, and e-mail kayeelee@ uchicago.edu.

Stacey A. Maskarinec is currently an $\mathrm{MD} / \mathrm{PhD}$ degree candidate in the UCLA/Caltech Medical Scientist Training Program. She received a BS degree in chemistry from the University of Chicago in 2002, where she authored several publications describing the interaction of triblock copolymers with lipid monolayers under the direction of Ka Yee C. Lee. She was the 2002 recipient of the F. \& I. Scherer Memorial Award for outstanding achievement in undergraduate research. In 2004, she joined the laboratory of David A. Tirrell to pursue her doctoral research in chemistry. Her primary focus is on the design of artificial proteins for biomaterials, for which she was awarded an NIH fellowship.

Maskarinec can be reached at Caltech, 1200 E. California Blvd., MC 210-41, Pasadena, CA, 91125 USA; tel. 626-3952511, fax 626-793-8472,

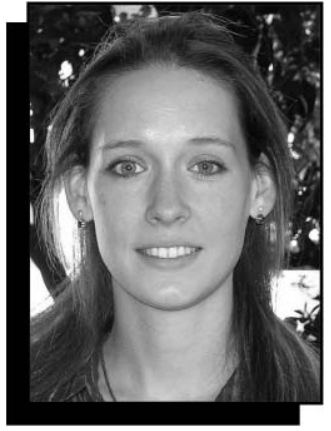

Stacey A. Maskarinec

and e-mail stacey@ caltech.edu.

Darryl Y. Sasaki is a staff scientist at Sandia National Laboratories in the Biomolecular Interfaces and Systems Department. He obtained his BS degree in chemistry from the University of Hawaii and his $\mathrm{PhD}$ degree in organic chemistry from the University of California, Irvine. He was a research associate at the ERATO Kunitake Project in Japan and at the California Institute of Technology. His current research interests are in self-assembled systems, molecular recognition, biomembrane structure, and sensor materials.

Sasaki can be reached at Sandia National Laboratories, PO Box 969, MS 9292, Livermore, CA 94551 USA; tel. 925-2942922, fax 925-294-3020, and e-mail dysasak@ sandia.gov.

Mark J. Stevens is a staff scientist at Sandia National Laboratories. He received a BS degree in physics and a BA degree in mathematics from the University of Cincinnati, and a PhD degree in physics from the Johns Hopkins University. His current research is focused on biomembranes, membrane proteins, charged polymers, adhesives, 


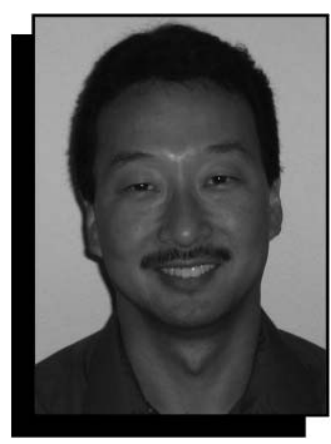

Darryl Y. Sasaki

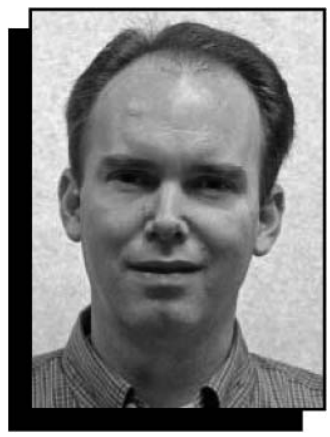

Brian Webb

biopolymer networks, and self-assembled monolayers.

Stevens can be reached at Sandia National Laboratories, PO Box 5800, MS 1411, Albuquerque, NM 87185 USA; tel. 505-8441937, fax 505-844-9781, and e-mail msteve@ sandia.gov.

\section{Motomu Tanaka re-} ceived his PhD degree from Kyoto University in 1998. He joined the group of Erich Sackmann in the Depart-

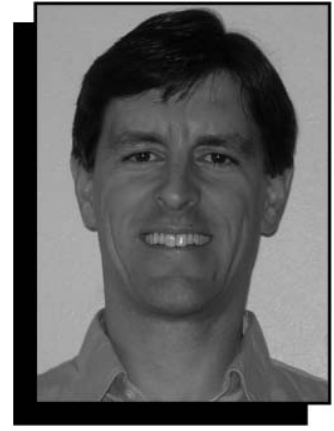

Mark J. Stevens

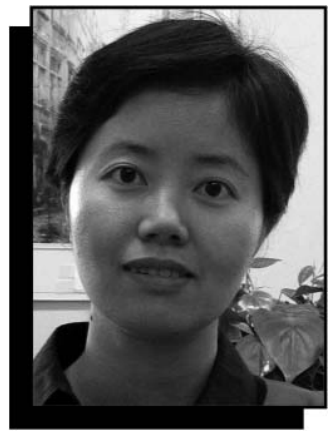

Guohui Wu

ment of Physics at the Technical University of Munich (TUM) as a fellow of both the Japan Society for the Promotion of Science and the Alexander von Humboldt Foundation. From 2001 to 2005 , he led an independent research group at the TUM as an Emmy Noether Fellow of the German Science Foundation (DFG) and received the habilitation degree in experimental physics from the TUM in 2005. He then became

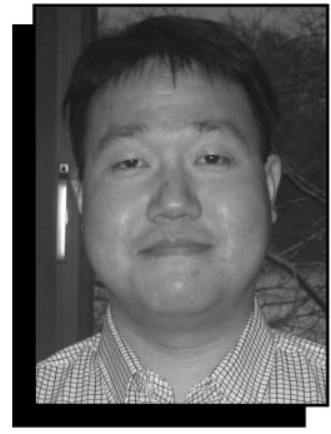

Motomu Tanaka

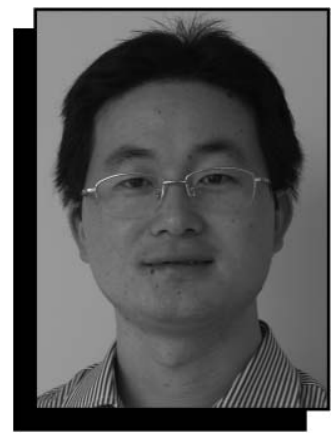

Liangfang Zhang

a professor of biophysical chemistry in the Institute of Physical Chemistry at the University of Heidelberg. His research interests cover physics of cells, biological membranes, and biopolymers and their applications in materials science.

Tanaka can be reached at the University of Heidelberg, Institute for Physical Chemistry, D69120 Heidelberg, Germany; e-mail tanaka@ uni-heidelberg.de.
Brian Webb is a senior research scientist at Corning Inc. He received a bachelor's degree in biochemistry from Brigham Young University in 1992 and a PhD degree in biochemistry from the University of Wisconsin-Madison in 1997. He then did a postdoctoral fellowship at the University of California, Berkeley, from 1997 to 2000 before joining Corning in 2000. His research interests include high content screening, cell-based assays, and protein microarray technologies.

Webb can be reached at Corning Inc., $1 \mathrm{Sci}-$ ence Center Drive, SPFR-01-5, Painted Post, NY 14870 USA; tel. 607974-9069 and e-mail webbbl@corning.com.

Guohui Wu is currently a postdoctoral research associate at the University of California, Santa Barbara. She received her PhD degree in physical chemistry from the University of Chicago in 2005. For her graduate work, she studied the interaction between lipid membranes and triblock copolymers.

Her current postdoctoral research involves developing biomaterials and nanoparticles as carriers for drug delivery. She received a Burroughs Wellcome Fund Interfaces fellowship and the Elizabeth $\mathrm{R}$. Norton Prize for Excellence in Research in Chemistry from the University of Chicago.

Wu can be reached at the University of California, Santa Barbara, Department of Chemical Engineering, Santa Barbara, CA 93106-5080 USA; tel. 805-893-4349 and e-mail guohuiwu@ engineering.ucsb.edu.

Liangfang Zhang is a graduate student in the Chemical and Biomolecular Engineering Department at the University of Illinois at Urbana-Champaign. He received his BS and MS degrees from the Chemical Engineering Department at Tsinghua

University in China in 2000 and 2002, respectively. Under the guidance of Steve Granick at UIUC, his current research involves dynamics of single lipids and proteins in lipid membranes, phases and phase transitions of biomembranes, and nanoparticleliposome complexes.

Zhang can be reached at the University of Illinois at UrbanaChampaign, Materials Research Laboratory, 104 South Goodwin Avenue, Urbana, IL 61801 USA; tel. 217-244-7386 and e-mail zhang6@ uiuc.edu.

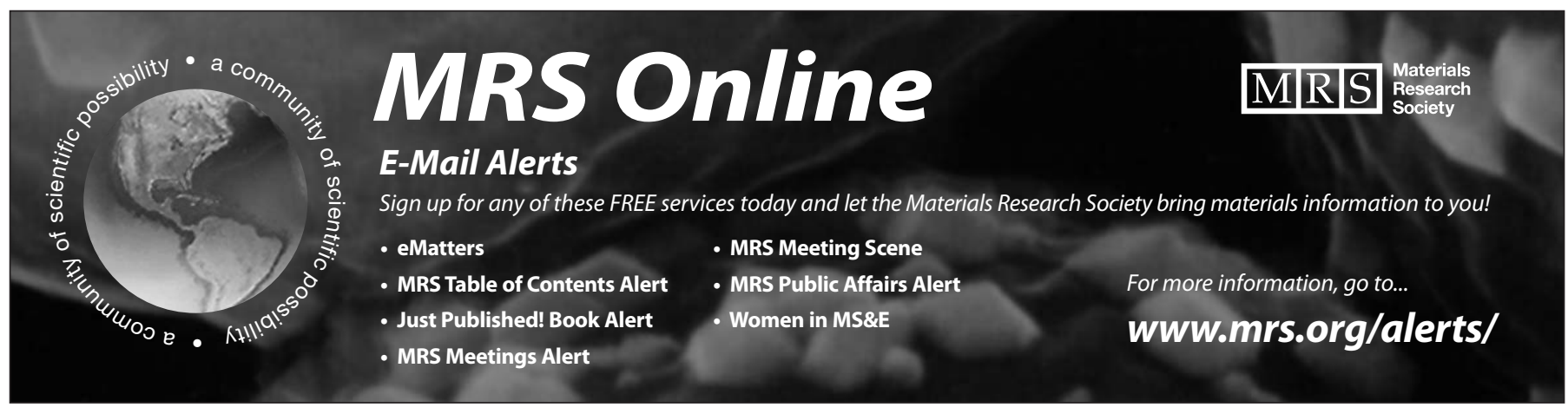

